\title{
Theoretical study of magnetic moments induced by defects at the $\mathrm{SiC}(110)$ surface
}

\author{
Adrien Poissier, ${ }^{1}$ Nicolás Lorente, ${ }^{2}$ and Félix Yndurain ${ }^{3}$ \\ ${ }^{1}$ Physics and Astronomy, State University of New York, Stony Brook, New York 11794-3800, USA \\ ${ }^{2}$ Centre d'Investigació en Nanociència i Nanotecnologia (CSIC-ICN), Campus de la Universitat Autònoma de Barcelona (UAB), \\ E-08193 Bellaterra, Spain \\ ${ }^{3}$ Departamento de Física de la Materia Condensada, Universidad Autónoma de Madrid, E-28049 Madrid, Spain
}

(Received 14 October 2010; published 24 January 2011)

\begin{abstract}
The effect of different surface defects on the atomic and electronic structures of cubic $\beta$-SiC(110) surface are studied by means of a first-principles calculation based on density-functional theory using the SIESTA code. In the calculations, different spin populations at each atom are allowed. We find that while adsorption of atomic $\mathrm{O}$, $\mathrm{N}$, or $\mathrm{H}$ on surface $\mathrm{C}$ atoms do not induce magnetic moments on $\mathrm{SiC}(110), \mathrm{Si}$ vacancies, substitutional $\mathrm{C}$ at the $\mathrm{Si}$ sites, and $\mathrm{H}$ or $\mathrm{F}$ adsorbed on $\mathrm{Si}$ surface sites induce localized magnetic moments as large as $0.7 \mu_{B}$ at the $\mathrm{C}$ atoms close to the defect. The local magnetic moment arrangement varies from ferromagnetic in the case of $\mathrm{H}$ adsorption to antiferromagnetic in the $\mathrm{Si}$ vacancy and substitutional $\mathrm{C}$ cases. The case of $\mathrm{H}$ adsorption on $\mathrm{Si}$ surface atoms is discussed in detail. It is concluded that magnetism is mainly owing to the local character of the $\mathrm{C}$ valence orbitals.
\end{abstract}

DOI: 10.1103/PhysRevB.83.035322

PACS number(s): 71.20.Mq, 75.70.Rf, 81.05.U-

The localized character of carbon valence charge has motivated the study of carbon atoms developing localized magnetic moments near defects. ${ }^{1}$ This has prompted the study of a variety of defects at different carbon atomic configurations such as graphite, ${ }^{2}$ graphene, ${ }^{3,4}$ nanotubes, ${ }^{5,6}$ and other configurations, ${ }^{7,8}$ including defect-free diamond surfaces. ${ }^{9}$ Here, in this work, we consider the appearance of localized magnetic moments near defects on the silicon carbide $(\beta$-SiC) (110) cubic surface. The quasi-one-dimensional character of the $\mathrm{Si}-\mathrm{C}$ chains at the surface and the localized character of the carbon atomic valence charge suggest the possibility of defect-induced magnetism.

For many years now, silicon carbide has been considered as a compound with important potential practical applications such as power electronics, heterogeneous catalysis support, structural and protective components for use in future nuclear fusion reactors, etc. Recently, this interest has been fostered by the possibility to obtain $\mathrm{SiC}$ in different atomic configurations ranging from macromolecules such as fullerenes to two-dimensional sheets and their wrapped configuration in nanotubes (see, for instance, the work of Melinon et al. ${ }^{10}$ and references therein).

The free $\mathrm{SiC}(110)$ surface has been theoretically studied by Sabisch et al. ${ }^{11}$ (for a complete review of the different $\mathrm{SiC}$ surfaces, see Ref. 12). Unlike other surface orientations, the free $\mathrm{SiC}(110)$ surface does not present any reconstruction, as different theoretical works reveal. ${ }^{12}$ To the best of our knowledge, there is no experimental study published about this surface.

Here, we present the results of a first-principles calculation of different defects at the $\mathrm{SiC}(110)$ surface. The calculations throughout this work were performed within the densityfunctional theory (DFT), ${ }^{13}$ using the generalized gradient approximation $(\mathrm{GGA})^{14}$ for the exchange and correlation potential. Our calculations have been obtained with the SIESTA ${ }^{15,16}$ method, which uses a basis set of numerical atomic orbitals ${ }^{17}$ and separable ${ }^{18}$ norm conserving pseudopotentials ${ }^{19}$ with partial core corrections. ${ }^{20}$ We have found the standard double- $\zeta$ basis with polarization orbitals (DZP) satisfactory, and it has been used throughout this work. The bulk calculation yields a lattice constant of $4.41 \AA$ (Si-C bond length of $1.91 \AA$ ), in fair agreement with the experimental value of $4.36 \AA$. We find a partially ionic Si-C bond with a charge transfer of 0.45 electrons to the $\mathrm{Si}$ atom as evaluated using a Mulliken population analysis. We obtain a band gap of $1.31 \mathrm{eV}$, similar to the $1.29 \mathrm{eV}$ band gap calculated by Sabisch et al. ${ }^{11}$ and to be compared to the experimental one of $2.417 \mathrm{eV}^{21}$

To simulate the free $\mathrm{SiC}(110)$ surface we have performed calculations of slabs formed by the stacking of (110) planes with different sizes. In particular, we have considered sevenand fifteen-layer slabs. In all cases, one side of the slab was properly saturated with hydrogen atoms in order to reproduce bulk behavior. The convergence of the relevant precision parameters was carefully checked. The real-space integration grid had a cutoff of 400 Ryd. Of the order of 600 $k$ points were used in the two-dimensional Brillouin zone by using the Monkhorst-Pack $k$-points sampling. To accelerate the self-consistency convergence, a polynomial broadening of the energy levels was performed using the Methfessel and Paxton's method. ${ }^{22}$ This method is known to be very suitable for systems with a large variation of the density of states at the vicinity of the Fermi level, which is the case here (see below). Broadening as Fermi-Dirac can be inappropriate and give the wrong results. It is worth mentioning that the energy differences between nonmagnetic and magnetic solutions are, in general, small, which requires a very high convergence in all precision parameters and tolerances. To obtain the equilibrium geometry, we relaxed the atoms until the forces acting on them were smaller than $0.01 \mathrm{eV} / \AA$.

Results on our calculations of a defect-free fifteen-layer slab are reported in Figs. 1 and 2. As in Ref. 11, we obtain a nonmetallic surface with two well-separated surface bands (see Fig. 1); the occupied narrow one is mainly owing to the $\mathrm{C}$ dangling bond, whereas the unoccupied wider one has a Si character, as the projected densities of states reveals (see Fig. 2). We, as Sabisch et al., ${ }^{11}$ obtain a buckled nonreconstructed 


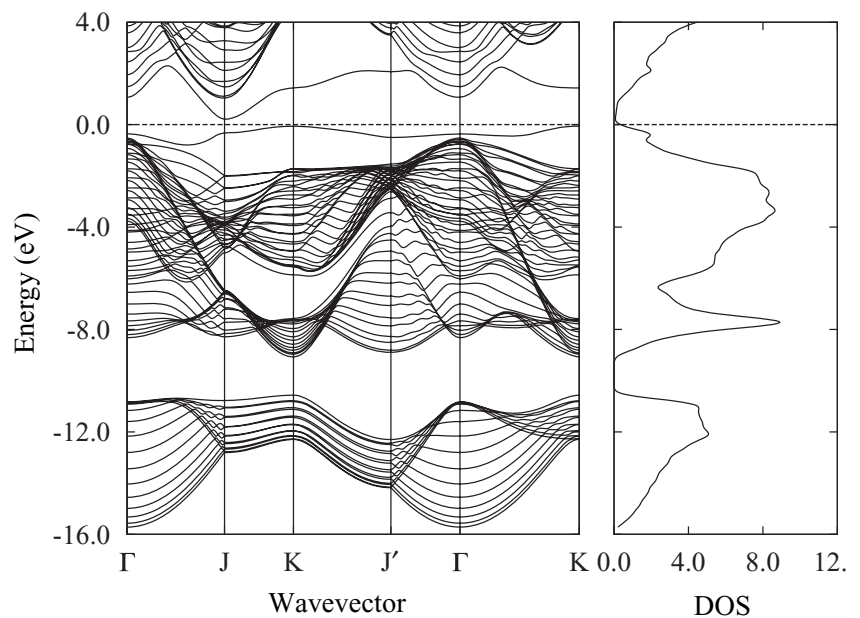

FIG. 1. Calculated band structure an total density of states for a fifteen-layer slab of $\mathrm{SiC}(110)$ with a buckled, unreconstructed clean surface. The surface-state band structure is apparent at the bulk band gap. The origin of energies is taken at the Fermi energy.

surface; the $\mathrm{C}$ and $\mathrm{Si}$ atoms are displaced normal to the surface by $0.01 \AA$ outward and by $0.24 \AA$ inward, respectively. Surface bonds are shorter $(1.81 \AA)$ than the bulk ones $(1.91 \AA)$ and bond angles $\left(\sim 122^{\circ}\right)$ at the surface are larger than at the bulk $\left(109.47^{\circ}\right)$, making the surface chains more one dimensional than the unrelaxed ones. We allowed in the calculation the possibility of different spin populations in the atoms. We did not find any stable solution with nonzero magnetic moment at the surface atoms. It is worth indicating that the densities of states in Fig. 2 suggest that introducing holes in the system would shift the Fermi level to the sharp C-like peak, giving rise to an eventual removal of states at the Fermi level by means of structural (Jahn-Teller type) and/or magnetic broken symmetries. However, introducing electrons would shift the Fermi level to a much wider Si-like band, with magnetic solutions being unlikely.

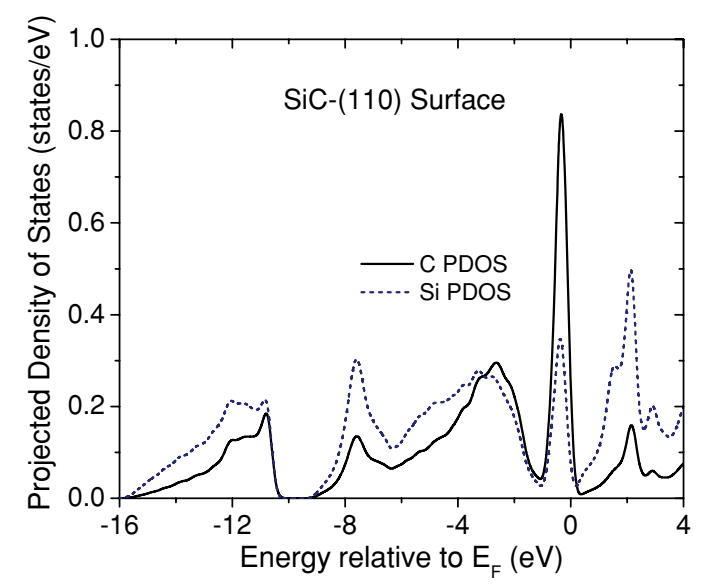

FIG. 2. (Color online) Projected electronic densities of states at the surface $\mathrm{C}$ (solid black line) and $\mathrm{Si}$ (dashed blue line) atoms in the $\mathrm{SiC}(110)$ surface. The origin of energies is taken at the Fermi energy. A small imaginary contribution to the energy has been added for presentation purposes.
TABLE I. Charge $(Q)$ and magnetic moment $(\mu)$ at carbon atoms near different defects at the $\mathrm{SiC}(110)$ surface. The charges are the Mulliken populations. $Q \uparrow, \downarrow$ stand for the spin-up and spin-down electronic charges, respectively.

\begin{tabular}{lccccc}
\hline \hline Atom & $Q \uparrow$ & $Q \downarrow$ & $Q_{\text {tot }}$ & $\mu\left(\mu_{B}\right)$ & Si-C $(\AA)$ \\
\hline H on top of Si & & & & & \\
Si bonded to H & 2.154 & 2.142 & 4.296 & 0.012 & - \\
C at Fig. 3(a) & 2.179 & 1.474 & 3.653 & 0.705 & 1.89 \\
C at Fig. 3(b) & 1.902 & 1.844 & 3.746 & 0058 & 1.85 \\
F on top of Si & & & & & \\
Si bonded to F & 2.204 & 2.178 & 4.382 & 0.026 & \\
C next to Si & 2.144 & 1.491 & 3.635 & 0.653 & 1.87 \\
C next to Si & 1.916 & 1.814 & 3.730 & 0.102 & 1.84 \\
Si vacancy & & & & & \\
C at Fig. 6(a) & 2.353 & 1.509 & 3.862 & 0.843 & - \\
C at Fig. 6(b) & 1.571 & 2.059 & 3.630 & -0.488 & - \\
C at Fig. 6(a) & 2.353 & 1.509 & 3.862 & 0.843 & - \\
Substrate C & & & & & \\
C next to extra C & 2.071 & 1.855 & 3.926 & 0.216 & - \\
Extra C & 1.911 & 2.025 & 3.936 & -0.114 & - \\
C next to extra C & 2.071 & 1.855 & 3.926 & 0.216 & - \\
\hline \hline
\end{tabular}

We next consider different defects at the surface. We have considered a $2 \times 2$ surface unit cell with one defect in it. Nine (110) layers were included in the slab calculation. In most

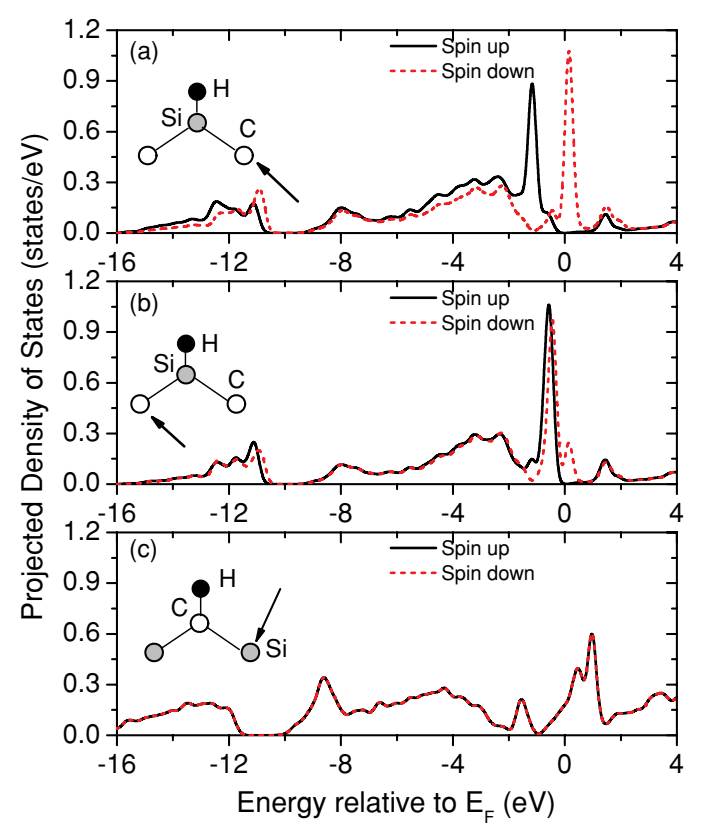

FIG. 3. (Color online) Projected spin-resolved densities of states at the vicinity of an hydrogen atom chemisorbed at the $\mathrm{SiC}(110)$ surface. Solid (dashed) lines represent spin-up (spin-down) states. (a) and (b) Densities of states projected on the $\mathrm{C}$ atom closest to the defect ( $\mathrm{H}$ bonded to the surface $\mathrm{Si}$ atom). (c) Densities of states projected on the $\mathrm{Si}$ atom closest to the defect (H bonded to the surface $\mathrm{C}$ atom). The arrow in the insets indicate the atom where the density of states is projected. The origin of energies is taken at the Fermi energy. A small imaginary contribution to the energy has been added for presentation purposes. 


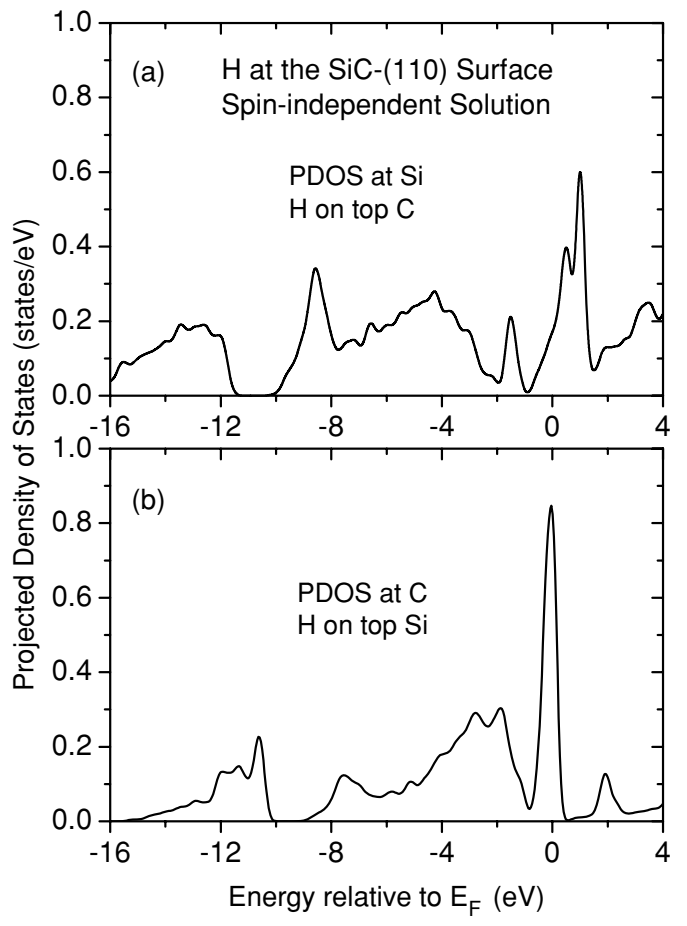

FIG. 4. Projected densities of states near adsorbed hydrogen in the spin-independent solution. (a) Projected density of states at the Si surface atom next to the carbon atom bonded to the hydrogen impurity. (b) Projected density of states at the $\mathrm{C}$ surface atom next to the silicon atom bonded to the hydrogen impurity. In both cases the origin of energies is taken at the Fermi energy.

cases, results were checked by enlarging the supercell size. We first study a hydrogen atom saturating either a $\mathrm{Si}$ or a $\mathrm{C}$ surface atom. In the case of hydrogen bonded to a $\mathrm{Si}$ atom, we obtain, after full geometry relaxation, a magnetic solution such that the surface carbon atoms next to the defect have magnetic moments of $0.705 \mu_{B}$ and $0.058 \mu_{B}$, respectively (see Table I). This magnetic moment is very localized at one of the carbon atoms, the other carbon atoms in the unit cell have a magnetic moment of the order of $0.02 \mu_{B}$, whereas the induced magnetic moments at the silicon atoms is also very small (see Table I). It should be indicated that the calculated total magnetic moment in the slab is $1 \mu_{B}$. This magnetic solution is the most stable one with an energy gain of $0.142 \mathrm{eV}$ per unit cell with respect to the diamagnetic one. Spin-resolved projected densities of states are shown in Fig. 3. The splitting of the carbon surface-state peak owing to the local magnetic moment is apparent. The magnetic moment symmetry of the two $\mathrm{C}$ atoms bonded to the defect is broken (see Table I) and, in addition, the symmetric geometry is also broken, including a buckling of $0.22 \AA$ of the $\mathrm{C}$ atoms. It is worth noticing that while the magnetic solution is accompanied by this Jahn-Teller-type distortion, a spin-independent calculation does not give rise to any structural distortion. Our result clearly indicates a coupling between lattice distortions and local magnetic moments.

The same calculations were performed in the case of $\mathrm{H}$ attached at the surface carbon atom. In this case, as anticipated, no magnetic stable solution was found, obtaining a paramagnetic ground state.
In order to understand the origin of the localized moments when $\mathrm{H}$ is bonded to $\mathrm{Si}$, and their absence when $\mathrm{H}$ is attached to $\mathrm{C}$, we present in Fig. 4 the results of the projected densities of a spin-independent calculation. We first observe that in both cases the Fermi level lies at a band and therefore the system is metallic. However, the main difference between $\mathrm{C}$ and $\mathrm{Si}$ is that the density of states at the Fermi level in the case of $\mathrm{H}$ on top of $\mathrm{Si}$ is much higher than in the case of $\mathrm{H}$ on top of $\mathrm{C}$. The $\mathrm{C}$ band is much narrower than the Si band owing to the more localized valence orbitals in $\mathrm{C}$ than in $\mathrm{Si}$, and the corresponding covalent radii are 0.77 and $1.17 \AA$ for $\mathrm{C}$ and $\mathrm{Si}$, respectively. This local character in the $\mathrm{C}$ case is also responsible for a larger electron-electron Hubbard-like interaction $U$, and therefore the fulfillment of the condition $U \times N\left(E_{F}\right)>1$, with $N\left(E_{F}\right)$ the substrate density of states at the Fermi energy, to develop localized magnetic moments in a Stoner-like approach. ${ }^{23}$

Other possible surface defects were considered in detail:

(i) A fluorine impurity: $\mathrm{F}$ behaves as $\mathrm{H}$, it saturates the $\mathrm{Si}$ dangling bond, and induces magnetism in the nearby $\mathrm{C}$ atoms [see Table I and Fig. 5(c)]. The magnetic moments and atomic relaxations induced by $\mathrm{F}$ are very similar to those induced by $\mathrm{H}$. As in the case of $\mathrm{H}$, the total magnetic moment induced in the crystal is $1 \mu_{B}$.

(ii) An oxygen impurity: Oxygen can saturate the surface dangling bonds but energetically is much more favorable to break the $\mathrm{Si}-\mathrm{C}$ surface bond rather than saturating the dangling

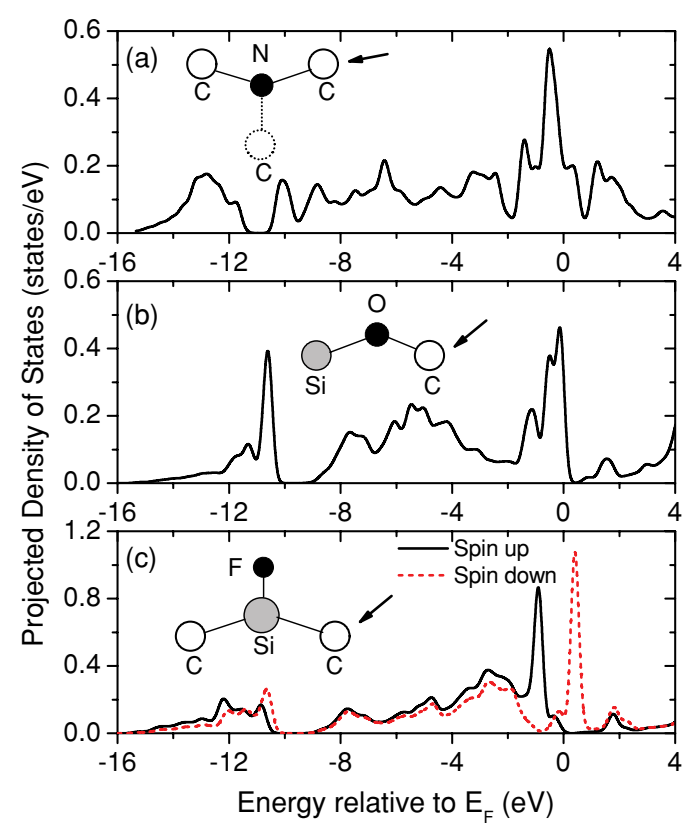

FIG. 5. (Color online) Projected densities of states near different impurities at the $\mathrm{SiC}(110)$ surface. The impurities are indicated by a black circle. The arrows indicate the $\mathrm{C}$ atoms where the density of states is projected. In the case of fluorine [(c)] both spin-up (continuous black) and spin-down (dashed red) densities of states are shown. In the case of oxygen [(b)] and nitrogen [(a)] there is no distinction between spin-up and spin-down bands. The atomic configurations at the different defect geometries are sketched. The dotted lines in (a) indicate the carbon atom under the topmost layer. 


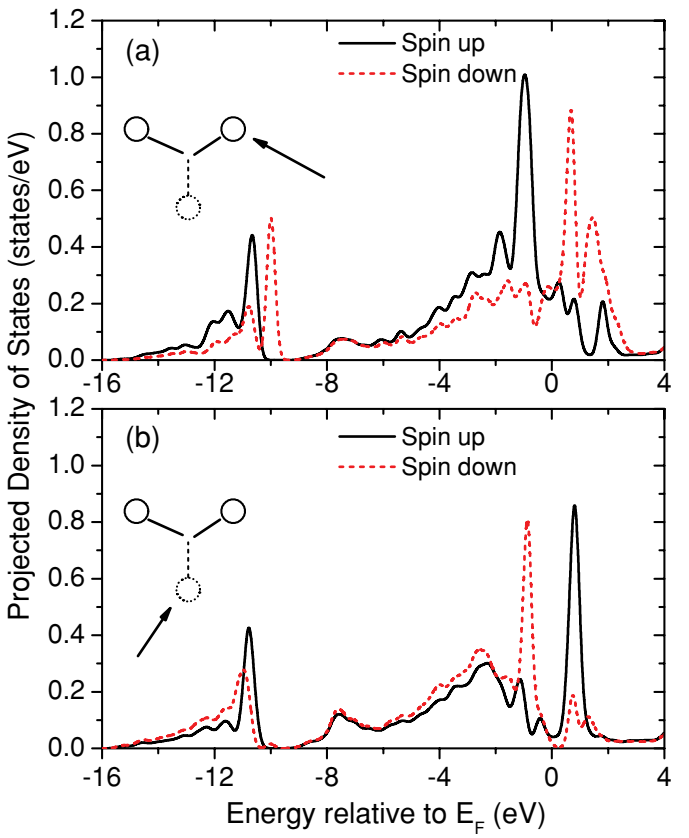

FIG. 6. (Color online) Projected densities of states near a $\mathrm{Si}$ vacancy at the $\mathrm{SiC}(110)$ surface. The surface $\mathrm{C}$ atoms are indicated by circles. The dotted lines the carbon atom under the topmost layer. The $\mathrm{C}$ atoms close to the vacancy where the densities of states are projected are indicated by an arrow. (a) Densities of states at the outermost $\mathrm{C}$ atoms. (b) Densities of states at the $\mathrm{C}$ atom at the underneath layer. Solid black (dashed red) lines stand for spin-up (spin-down) densities of states.

bonds, as in the formation of $\mathrm{SiO}_{2}$ at the $\mathrm{Si}$ surface. ${ }^{24}$ In this case, no magnetic solution was found. The corresponding projected density of states is drawn in Fig. 5(b). (iii) A substitutional nitrogen atom: The most stable configuration of a $\mathrm{N}$ impurity is by substituting a $\mathrm{Si}$ surface atom and being bonded to three $\mathrm{C}$ atoms. Results of the calculation of this defect display no magnetic moment. The density of states at one of the surface carbon atoms bonded to $\mathrm{N}$ is shown in Fig. 5(a).

(iv) A silicon vacancy: This configuration favors a magnetic solution at the $\mathrm{C}$ atoms (see Table I) such that the magnetic moment at the underneath carbon atom is in an antiferromagnetic configuration with respect to the two surface $\mathrm{C}$ atoms. The total magnetic moment in the slab is zero. The projected densities of states at the carbon atoms close to the vacancy are shown in Fig. 6

(v) Substitutional carbon at the surface layer: In this case we also obtain a magnetic solution at the carbon atom near the defect. The magnetic configuration of the carbon atoms is antiferromagnetic as in the case of the C-(110) surface $^{9}$. The total magnetic moment in the slab is also zero.

In conclusion, we have found that different defects at the $\mathrm{SiC}(110)$ surface can induce localized magnetic moments at $\mathrm{C}$ atoms near the defect with various magnetic configurations. In the case of hydrogen or fluorine adsorbed at a Si surface atom, magnetism is accompanied by a local lattice distortion. Magnetism takes place mainly at the carbon atoms owing to the localized character of its atomic valence charge. We have found that the $\mathrm{SiC}(110)$ surface is an excellent candidate for magnetic configurations owing to the quasi-one-dimensional behavior of the topmost $\mathrm{Si}-\mathrm{C}$ chains.

We thank J. M. Soler for helpful discussions. Financial support of the Spanish Ministry of Science and Innovation through Grants No. FIS2009-12712 and No. CSD2007-00050 is acknowledged.
${ }^{1}$ T. L. Makarova, B. Sundqvist, R. Höhne, P. Esquinazi, Y. Kopelevich, P. Scharff, V. A. Davydov, L. S. Kashevarova, and A. V. Rakhmanina, Nature (London) 413, 716 (2001).

${ }^{2}$ M. M. Ugeda, I. Brihuega, F. Guinea, and J. M. Gomez-Rodriguez, Phys. Rev. Lett. 104, 096804 (2010).

${ }^{3}$ J. Nilsson, A. H. Castro Neto, N. M. R. Peres, and F. Guinea, Phys. Rev. B 73, 214418 (2006).

${ }^{4}$ S. Choi, B. W. Jeong, S. Kim, and G. Kim, J. Phys. Condens. Matter 20, 235220 (2008).

${ }^{5}$ Y. Ma, P. O. Lehtinen, A. S. Foster, and R. M. Nieminen, Phys. Rev. B 72, 085451 (2005).

${ }^{6}$ P. O. Lehtinen, A. S. Foster, Y. Ma, A. V. Krasheninnikov, and R. M. Nieminen, Phys. Rev. Lett. 93, 187202 (2004).

${ }^{7}$ R. Höhne and P. Esquinazi, Adv. Mater. (Weinheim, Ger.) 14, 753 (2002).

${ }^{8}$ P. Esquinazi and R. Höhne, J. Magn. Magn. Mater. 290-291, 20 (2005).

${ }^{9}$ F. Yndurain, Phys. Rev. B 75, 195443 (2007).

${ }^{10}$ P. Melinon, B. Masenelli, F. Tournus, and A. Perz, Nat. Mater. 6, 479 (2007).

${ }^{11}$ M. Sabisch, P. Krüger, and J. Pollmann, Phys. Rev. B 51, 13367 (1995).
${ }^{12}$ J. Pollmann and P. Krüger, J. Phys. Condens. Matter 16, S1659 (2004).

${ }^{13}$ W. Kohn and L. J. Sham, Phys. Rev. 140, A1133 (1965).

${ }^{14}$ J. P. Perdew, K. Burke, and M. Ernzerhof, Phys. Rev. Lett. 77, 3865 (1996).

${ }^{15}$ J. M. Soler, E. Artacho, J. D. Gale, A. Garcia, J. Junquera, P. Ordejón, and D. Sánchez-Portal, J. Phys. Condens. Matter 14, 2745 (2002).

${ }^{16}$ P. Ordejón, E. Artacho, and J. M. Soler, Phys. Rev. B 53, R10441 (1996).

${ }^{17}$ O. F. Sankey and D. J. Niklewski, Phys. Rev. B 40, 3979 (1989).

${ }^{18}$ L. Kleinman and D. M. Bylander, Phys. Rev. Lett. 48, 1425 (1982).

${ }^{19}$ N. Troullier and J. L. Martins, Phys. Rev. B 43, 1993 (1991).

${ }^{20}$ S. G. Louie, S. Froyen, and M. L. Cohen, Phys. Rev. B 26, 1738 (1982).

${ }^{21}$ W. R. L. Lambrecht, B. Segall, M. Yoganathan, W. Suttrop, R. P. Devaty, W. J. Choyke, J. A. Edmond, J. A. Powell, and M. Alouani, Phys. Rev. B 50, 10722 (1994).

${ }^{22}$ M. Methfessel and A. T. Paxton, Phys. Rev. B 40, 3616 (1989).

${ }^{23}$ P. W. Anderson, Phys. Rev. 124, 41 (1961).

${ }^{24}$ E. Martinez, J. Plans, and F. Yndurain, Phys. Rev. 36, 8043 (1987). 